\title{
Cenizas del tamo de arroz como substituto del feldespato en la fabricación de cerámica blanca
}

\author{
ÁlVARO GUZMÁN A ${ }^{* *}$, SILVIO DELVASTO A', ENRIQUE SÁNCHEZ V², VICENTE AMIGÓ B ${ }^{3}$ \\ 1: Grupo de Investigación de Materiales Compuestos, GMC. Escuela de Ingeniería de Materiales, Universidad del Valle, Cali, Colombia \\ 2: Instituto de Tecnología Cerámica, Universidad Jaume I, Castellón, España \\ 3: Instituto de Tecnología de Materiales, Universidad Politécnica de Valencia, Valencia, España \\ *alvaro8308@hotmail.com
}

\begin{abstract}
La sustitución de materias primas para procesamiento de materiales -de alto consumo energético- por residuos agrícolas y agroindustriales impacta positivamente el medio ambiente. Uno de estos residuos es la paja o tamo de arroz, del cual la FAO estima que su disponibilidad mundial ronda los 600 millones de toneladas por año. En éste artículo se presentan los resultados de una investigación sobre la utilización de una ceniza de tamo o paja de arroz (CTA) como substituto del feldespato en la fabricación de cerámica blanca de tipo triaxial. Se prepararon mezclas donde la ceniza sustituyó al feldespato en volúmenes distintos. Especímenes de las pastas obtenidas fueron moldeados, secados, y cocidos. Se evaluaron las propiedades físicas y mecánicas de las cerámicas cocidas. La porosidad y la resistencia a la compresión de las piezas cocidas aumentan con adiciones de CTA de hasta un $75 \%$ en reemplazo del feldespato. Mediante DRX se estudió la evolución de las fases debido a la adición de CTA a la composición de la porcelana tradicional, y por MEB se estudió la microestructura, de granos de cuarzo y agujas de mullita primaria y secundaria en una matriz vítrea feldespática. Se concluyó que la CTA bajo las condiciones de obtención, sí reemplaza parcialmente al feldespato en la elaboración de pastas de loza.
\end{abstract}

Palabras Clave: mezcla triaxial, cerámica blanca, porcelana, tamo de arroz, ceniza de tamo de arroz, feldespato.

\section{Use of rice straw ash as substitute of feldspar in triaxial porcelain}

The substitution of raw materials for processing high energy consumption materials by agricultural and agro-industrial wastes causes a positive impacts on the environment preservation. One of these residues is rice straw, which according to FAO estimation, its annual production is about 600 million tons. In this research was studied the use of rice straw ash (RSA) as substitute of the use of feldspar in the whiteware production. Clay-feldspar-quartz porcelains are referred to as triaxial whiteware. Specimens of semidry triaxial mixtures, where feldspar was substituted for different percentages of CTA, were prepared by uniaxial pressing, followed by drying and sintering. Physical and mechanical properties of sintered bodies were evaluated. The porosity and the compressive strength of the fired pieces do increase with additions of up to $75 \%$ of CTA in substitution of feldspar. Their mineralogical phases were determined by DRX and SEM; grains of quartz, and needles of primary and secondary mullite were identified in a vitreous phase. It was concluded that feldspar can be substituted positively by CTA in whiteware pastes.

Keywords: Triaxial mixes, whiteware, porcelain, rice straw, rice straw ash, feldspar.

\section{INTRODUCCIÓN}

La producción anual de tamo (TA) o pajilla de arroz (rice straw) según la FAO (Food and Agriculture Organization) se estima en cerca de los 600 millones de toneladas por año [1]. El TA es quemado frecuentemente al aire libre, causando polución, sobrecalentamiento, degradación del suelo y contaminación del agua [2] y, también es incorporado al suelo como abono orgánico o usado como forraje para los animales [3]; su uso directo en la alimentación animal es limitado dada su baja digestibilidad, ya que el tamo se encuentra constituido fundamentalmente por celulosa $(32-47 \%)$ y por lignina (5 - 24\%), componentes que son de difícil biodegradación $[1,4]$; en aras a mejorar la digestibilidad del mismo, han surgido soluciones para mejorar su uso en la alimentación animal, como lo son complementar la dieta de los animales con suplementos correctivos ó realizar tratamientos del tamo con urea (amonificación), hidróxido de sodio ó hidróxido de amonio [5]. Diversos autores mencionan el uso del TA como fuente de fibras y celulosa para la producción de pulpa y papel, y como materia prima para la producción de paneles de tableros de densidad media (medium density fiberboard (MDF)) $[5,6,7]$.

En Colombia el arroz es el tercer producto agrícola, después del café y del maíz; y representa el 13\% del área cosechada, 490.000 hectáreas, y el 30\% de los cultivos transitorios. Su producción representa el $6 \%$ del valor de la producción agropecuaria y el $11 \%$ de la actividad agrícola colombiana; generando aproximadamente 6 toneladas de arroz paddy/ hectárea cosechada $[2,3,8,9]$. Por su parte, el TA, es un 
desecho resultante de la cosecha de éste cereal constituido por el tallo, raíz y parte de las espigas de la planta. En Colombia se dispondrían de 4.98 toneladas de tamo/hectárea cultivada para un total de 2.5 millones de toneladas de tamo, si se toman los resultados de un estudio realizado en Hanoi (Vietnam), el cual indica que en promedio $0.83 \mathrm{~kg}$ de tamo es producido por cada kilogramo de arroz paddy [10].

Por otra parte, en general, las pastas de cerámica fina que se fabrican son de tipo triaxial, lo que significa que los principales ingredientes son la arcilla, el feldespato y un material de relleno (usualmente cuarzo o alúmina). Estas materias primas se pueden extraer de la naturaleza puras y a bajo coste; las pastas que forman son manejables y a partir de ellas se obtienen cerámicas cocidas con una estructura apropiada [11, 12, 13]. Comúnmente, las porcelanas de arcillafeldespato-cuarzo son conocidas como lozas blancas triaxiales. En Colombia, el feldespato es una materia prima costosa y su reemplazo representaría una significante reducción en los costos finales de la producción de porcelana [14, 15]. El feldespato potásico es el mineral fundente comúnmente usado en las pastas de porcelana patrón; sin embargo, las fuentes de minerales feldespáticos de alto grado recientemente han empezado a escasear, siendo así necesario pensar en una fuente alternativa de materiales fundentes para pasta de porcelana, el cual pueda servir a temperatura más baja en la cual el líquido viscoso se forme [16]. En Colombia, el feldespato potásico normalmente se importa, siendo por tanto, atractiva la posibilidad de sustituirlo por CTA, debido a que el contenido de $\mathrm{K}_{2} \mathrm{O}$ en cenizas de tamo de arroz se ha llegado a encontrar en el orden de 11,30 - 12,30\% [17, 18].

\section{MATERIALES Y MÉTODOS}

\subsection{Materiales}

El tamo de arroz estudiado corresponde a la variedad de arroz Fedearroz 60 (FL 3188). La recolección fue realizada de un cultivo localizado en Jamundí (Valle del Cauca, Colombia) a 1000 m.s.n.m. Las demás materias primas utilizadas para la elaboración de las pastas cerámicas triaxiales fueron cuarzo, feldespato y caolín, que son de origen colombiano.

La composición química y las principales fases cristalinas de las materias primas fueron determinadas mediante las técnicas de Fluorescencia de Rayos X (FRX) y Difracción de Rayos X (DRX), respectivamente (ver tabla I y figura 1). Los resultados mostraron en el caolín picos de gran intensidad a ángulos $2 \theta=20,87^{\circ}$ y $26,64^{\circ}$ correspondientes al cuarzo $\left(\mathrm{SiO}_{2}\right)(\mathrm{ICSD} 83849), 2 \theta=12,39^{\circ}$ y $59,9^{\circ}$ correspondientes a la caolinita $\left.\left(\mathrm{Al}_{2}\left(\mathrm{Si}_{2} \mathrm{O}_{5}\right)(\mathrm{OH})_{4}\right)\right)(\mathrm{ICSD} 87771)$, y $2 \theta=8,86 ; 19,88^{\circ}$; $22,87^{\circ}$ y $34,99^{\circ}$ correspondientes a la moscovita $2 \mathrm{M} 1 \mathrm{KAl}_{2}$

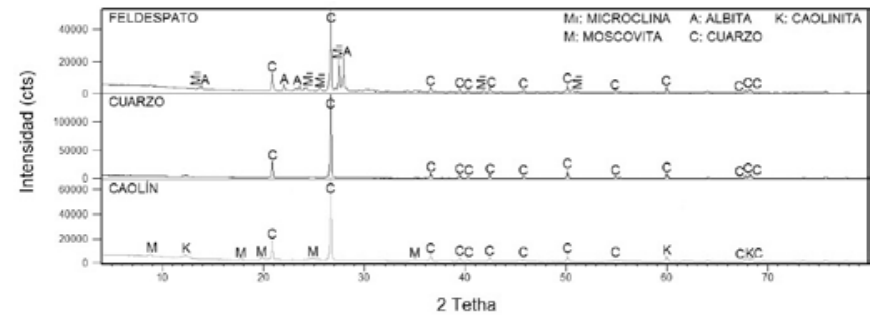

Figura 1. Patrones DRX de las materias primas.

$\left.\left(\left(\mathrm{AlSi}_{3}\right) \mathrm{O}_{10}\right)(\mathrm{OH})_{2}\right)$ (ICSD 17049). Por otra parte, el feldespato reveló picos de gran intensidad a ángulos $2 \theta=20,87^{\circ}$ y $26,64^{\circ}$ correspondientes al cuarzo $\left(\mathrm{SiO}_{2}\right)$ (ICSD 83849), $2 \theta=24,75^{\circ}$; $25,66^{\circ}$ y $27,47^{\circ}$ correspondientes a la microclina $\left(\mathrm{K}\left(\mathrm{AlSi}_{3} \mathrm{O}_{8}\right)\right.$ ) (ICSD 35334), y $2 \theta=22,05^{\circ} ; 23,57^{\circ}$ y $27,93^{\circ}$ correspondientes a la albita $\left(\left(\mathrm{Na}_{0.98} \mathrm{Ca}_{0.02}\right)\left(\mathrm{Al}_{1.02} \mathrm{Si}_{2.98} \mathrm{O}_{8}\right)\right)$ (ICSD 90142); mientras el cuarzo evidenció picos de gran intensidad a ángulos $2 \theta=$ $20,87^{\circ} ; 26,65^{\circ}$ y $50,14^{\circ}$ correspondientes al cuarzo $\left(\mathrm{SiO}_{2}\right)$ (ICSD 83849). Los diámetros medios de partícula del caolín, feldespato y cuarzo fueron $25,98 \mu \mathrm{m}, 18,56 \mu \mathrm{m}$ y $19,79 \mu \mathrm{m}$, respectivamente; el tamaño de partícula del cuarzo está entre 15 y $30 \mu \mathrm{m}$, dentro del rango recomendado por la literatura para la máxima resistencia de la porcelana [11, 19, 20, 21].

\subsection{Obtención y Caracterización de la Ceniza de Tamo de Arroz}

Se hizo una combustión imperfecta o quema del TA en un horno, con el fin de retirar el mayor contenido de materia orgánica presente en el material a una temperatura por encima del punto de inflamación, que es la temperatura a la cual el tamo como combustible es capaz de proseguir por sí solo la combustión, una vez que ésta se haya iniciado; aquí la temperatura máxima de llama fue de $497{ }^{\circ} \mathrm{C}$ y se obtuvo tamo de arroz carbonizado (TAC). Obtenido el TAC se realizó una combustión controlada de éste en un horno eléctrico para obtener cenizas de tamo de arroz (CTA) siguiendo los siguientes procesos de calcinación: Proceso A $\left(500^{\circ} \mathrm{C}\right.$, máximo), Proceso B $\left(600^{\circ} \mathrm{C}\right.$, máximo), Proceso $\mathrm{C}$ $\left(700^{\circ} \mathrm{C}\right.$, máximo), Proceso $\mathrm{D}\left(800^{\circ} \mathrm{C}\right.$, máximo), Proceso $\mathrm{E}$ $\left(900^{\circ} \mathrm{C}\right.$, máximo) y Proceso $\mathrm{F}\left(1000^{\circ} \mathrm{C}\right.$, máximo). El tiempo de residencia en el horno a la máxima temperatura fue de 2 horas. Una vez obtenida la CTA fue llevada a una finura similar a la del feldespato mediante molienda de una hora en un molino de bolas de laboratorio. Posteriormente, se determinó la distribución granulométrica de la CTA molida y el feldespato mediante un equipo Láser Mastersizer 2000. Adicionalmente, se determinó el peso específico siguiendo el procedimiento descrito en la norma ASTM C188, en forma

TABLA I. COMPOSICIÓN QUÍMICA DE LAS MATERIAS PRIMAS.

\begin{tabular}{|c|c|c|c|c|c|c|c|c|c|c|c|}
\hline \multirow{2}{*}{ MATERIA PRIMA } & \multicolumn{11}{|c|}{ Compuesto y/o elemento } \\
\hline & $\mathrm{SiO}_{2}$ & $\mathrm{TiO}_{2}$ & $\mathrm{Al}_{2} \mathrm{O}_{3}$ & $\mathrm{Fe}_{2} \mathrm{O}_{3}$ & $\mathrm{Mn}_{3} \mathrm{O}_{4}$ & $\mathrm{MgO}$ & $\mathrm{CaO}$ & $\mathrm{Na}_{2} \mathrm{O}$ & $\mathrm{K}_{2} \mathrm{O}$ & $\mathrm{P}_{2} \mathrm{O}_{5}$ & PF \\
\hline Caolín & 69,86 & 0,07 & 19,97 & 1,32 & - & 0,26 & - & 0,04 & 2,92 & - & 5,50 \\
\hline Cuarzo & 94,45 & 0,09 & 3,93 & 0,11 & - & - & - & - & - & - & 1,54 \\
\hline Feldespato & 76,12 & 0,07 & 13,61 & 0,27 & - & - & 0,40 & 3,51 & 5,38 & - & 0,68 \\
\hline
\end{tabular}


análoga al cemento. Su composición química fue determinada mediante Fluorescencia de Rayos X (FRX) en un espectrómetro MagixPro PW - 2440 Philips. El porcentaje de pérdida al fuego de la ceniza fue determinado de manera análoga al cemento, haciendo uso de la norma ASTM C114. La composición mineralógica de la CTA fue obtenida mediante la técnica de Difracción de Rayos X (DRX).

\subsection{Evaluación de la CTA como substituto del feldespato en la fabricación de cerámica blanca}

Mezclas de pastas triaxiales fueron preparadas con $50 \%$ de caolín, $25 \%$ de cuarzo y $25 \%$ de feldespato (CTA $0 \%$ ). Se prepararon cuatro mezclas más, en las cuales la proporción de feldespato fue reemplazada en un 25, 50, 75 y 100\% por la CTA (CTA 25\%, CTA 50\%, CTA 75\% y CTA 100\%). 1 kg por cada mezcla fue preparado por molienda en seco mediante el uso de un molino de bolas, utilizando como medio de molienda cuerpos moledores de alúmina con geometría cilíndrica durante un tiempo de 30 minutos. $20 \mathrm{~g}$ de cada mezcla semiseca ( $8 \%$ agua) fueron prensados uniaxialmente en un molde de acero a $80 \mathrm{MPa}$ en especímenes de $2,5 \mathrm{~mm}$ x 50,0 mm. Los especímenes fueron secados en un horno mufla a $110{ }^{\circ} \mathrm{C}$ y posteriormente cocidos a una temperatura máxima de $1200{ }^{\circ} \mathrm{C}$ con un tiempo de sostenimiento de 24 horas a la máxima temperatura en un horno eléctrico de laboratorio MTI KSL 1600X. A las probetas obtenidas, les fue evaluado el grado de vitrificación mediante la realización de ensayos de contracción lineal, porosidad, absorción y densidad conforme a las normas ASTM C326-09, ASTM C329-88 y ASTM C373-88. Finalmente, la resistencia a la compresión fue determinada de manera análoga a la norma ASTM C773-88 a una velocidad de $1 \mathrm{~mm} / \mathrm{min}$, un promedio de tres mediciones fue tomado. A la mezcla patrón (CTA 0\%) y a aquella con CTA en reemplazo de feldespato que presentó la mejor resistencia mecánica les fueron identificadas las principales fases cristalinas por DRX mediante un difractómetro de rayos X PANalytical $X^{\prime}$ Pert PRO. Las características microestructurales fueron observadas mediante Microscopía Electrónica de Barrido (MEB), para ello la superficie de fractura de cada muestra fue pulida y atacada con una solución de ácido fluorhídrico (HF) al 5\% durante $3 \mathrm{~min}$, luego fue lavada con agua destilada y alcohol etílico, para posteriormente secarla y recubrirla con $\mathrm{Au}-\mathrm{Pd}$.

\section{RESULTADOS Y DISCUSIONES}

\subsection{Caracterización de la ceniza obtenida}

El patrón de difracción (DRX) obtenido para la ceniza producida a partir del proceso $B$, presentó picos de gran intensidad a ángulos $2 \theta=28,27^{\circ}$ y $40,45^{\circ}$ correspondientes a la silvita $(\mathrm{KCl})$ (ICSD 240503), y $2 \theta=21,74^{\circ}$ correspondiente a la cristobalita baja $\left(\mathrm{SiO}_{2}\right)$ (ICSD 34933) (ver figura 2). Thy, et al. (2006) mencionan que la silvita frecuentemente es observada luego de la quema del tamo de arroz y de otras gramíneas [18]. El patrón de difracción de la ceniza producida a partir del proceso E, denotó picos de gran intensidad a ángulos $2 \theta=21,76^{\circ}$ y $36,00^{\circ}$ correspondientes a la cristobalita $\left(\mathrm{SiO}_{2}\right)(\mathrm{ICSD} 34933)$ y $2 \theta=20,61^{\circ}$ correspondiente a la tridimita $\left(\mathrm{SiO}_{2}\right)$ (ICSD 1109) (ver figura 2).

La composición química por FRX de las CTA obtenidas mediante los procesos B y E permitió observar que los componentes principales fueron $\mathrm{SiO}_{2}$ y $\mathrm{K}_{2} \mathrm{O}$ (ver tabla II), corroborando los resultados reportados por Jenkins, et al. (1998); Thy, et al. (2006) [17, 18].

Con base en los resultados de FRX se decidió utilizar la ceniza obtenida a partir del proceso B, ya que esta presentaba un contenido elevado de $\mathrm{K}_{2} \mathrm{O}$ en comparación con aquella obtenida a partir del proceso E. Además, un proceso de calcinación a $600{ }^{\circ} \mathrm{C}$, prácticamente puede llevarse en condiciones donde el combustible es la biomasa del material del cual proviene la ceniza.

Esto es más amigable con el medio ambiente a una combustión donde se requiera combustible suplementario para alcanzar el nivel de temperatura requerido.

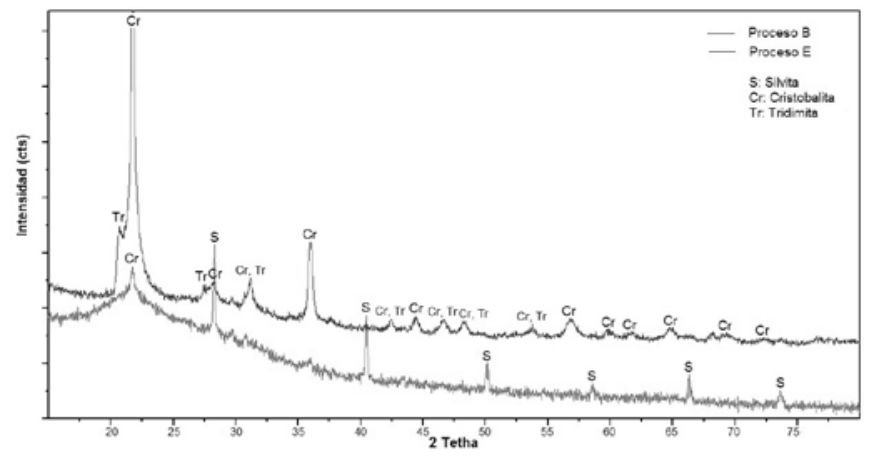

Figura 2. Patrones DRX para las CTA obtenidas de los procesos B y E.

TABLA II. COMPOSICIÓN QUíMICA CTA OBTENIDAS A PARTIR DE LOS PROCESOS B Y E.

\begin{tabular}{|c|c|c|c|c|c|c|c|c|c|c|c|}
\hline \multirow{2}{*}{ PROCESO } & \multicolumn{11}{|c|}{ Compuesto y/o elemento } \\
\hline & $\mathrm{SiO}_{2}$ & $\mathrm{~K}_{2} \mathrm{O}$ & $\mathrm{CaO}$ & $\mathrm{Cl}$ & $\mathbf{P}_{2} \mathrm{O}_{5}$ & $\mathrm{MnO}$ & $\mathrm{MgO}$ & $\mathrm{S}$ & $\mathrm{Fe}_{2} \mathrm{O}_{3}$ & $\mathrm{Al}_{2} \mathrm{O}_{3}$ & $\mathrm{Na}_{2} \mathrm{O}$ \\
\hline Proceso B (\%) & 69,09 & 12,56 & 4,31 & 2,88 & 1,96 & 0,95 & 0,90 & 0,27 & 0,25 & 0,13 & 0,10 \\
\hline Proceso E $(\%)$ & 85,56 & 6,68 & 3,44 & - & 1,76 & 0,77 & 0,65 & 0,08 & 0,37 & 0,28 & 0,23 \\
\hline \multirow{2}{*}{ PROCESO } & \multicolumn{11}{|c|}{ Compuesto y/o elemento } \\
\hline & Zn & $\mathbf{R b}$ & $\mathrm{Br}$ & $\mathbf{N i}$ & $\mathrm{Sr}$ & $\mathrm{Cu}$ & $\mathbf{Y}$ & Ва & $\mathrm{Pb}$ & $\mathrm{TiO}_{2}$ & PF \\
\hline Proceso B $(\%)$ & 0,02 & 0,02 & 0,01 & 0,01 & 0,01 & 0,00 & - & - & - & - & 6,56 \\
\hline Proceso E (\%) & 0,02 & 0,01 & - & 0,01 & 0,01 & 0,01 & 0,00 & 0,04 & 0,01 & 0,03 & 0,03 \\
\hline
\end{tabular}




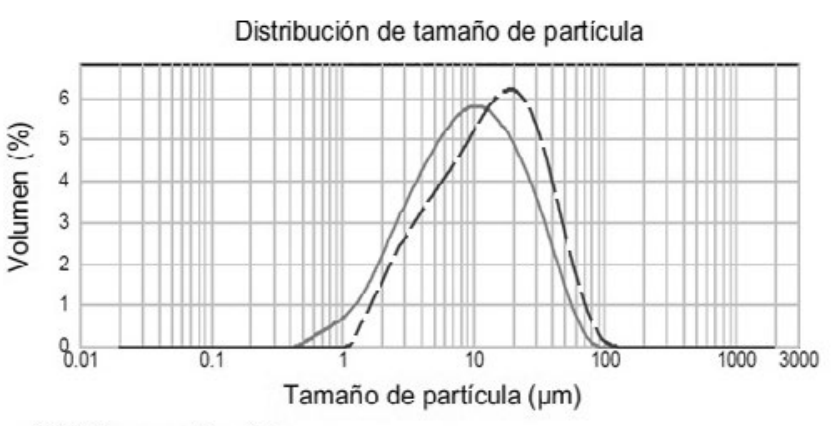

- CTA Proceso B molida

-- Feldespato

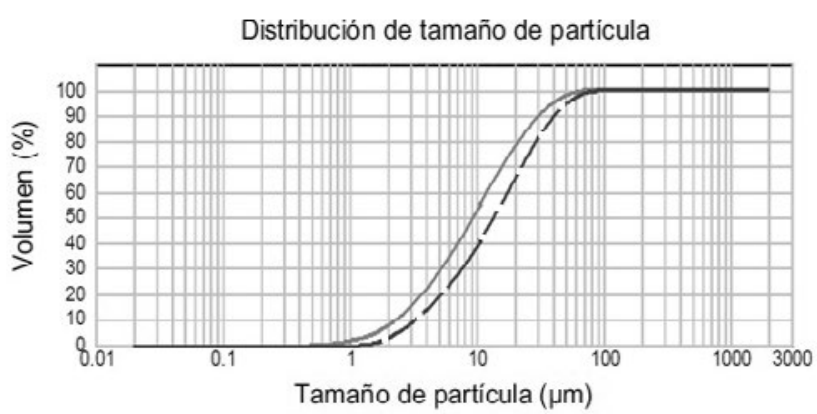

- CIA Proceso B molida

-- Feldespato

Figura 3. Distribución granulométrica CTA proceso B molida y feldespato.

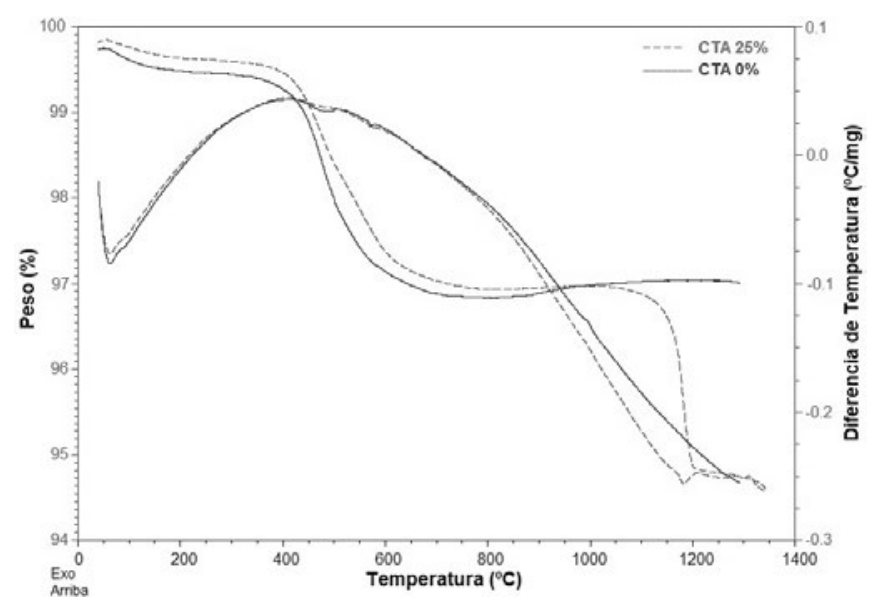

Figura 4. Curvas de ATD/ATG de mezclas de polvos de composición de porcelana dura cuyas pastas fueron dosificadas con $0 \%$ de CTA y con $25 \%$ de CTA.
La distribución de los tamaños acumulados de la CTA proceso B molida y del feldespato, resultaron ser similares (ver figura 3), y su diámetro medio de partícula D $(4,3)$ se encontró entre los 10 $20 \mu \mathrm{m}$. El peso específico de la ceniza fue de $2,28 \mathrm{~g} / \mathrm{cm}^{3}$.

\subsection{Evaluación de la aptitud como fundente de la CTA en la mezcla triaxial}

Los ensayos de ATD/ATG de las mezclas de polvos de composición de porcelana dura cuyas pastas fueron dosificadas con $0 \%$ de CTA y con $25 \%$ de CTA, mostraron un pico endotérmico en el rango de $0-200{ }^{\circ} \mathrm{C}$ atribuible a la remoción de agua adsorbida, seguido por la deshidroxilación de caolín (480 ${ }^{\circ} \mathrm{C}$ para CTA $0 \%$ y $470{ }^{\circ} \mathrm{C}$ para CTA $25 \%$ ) (ver figura 4 ). Aproximadamente a $572{ }^{\circ} \mathrm{C}$ se observó una banda endotérmica asociada a la transformación de cuarzo $\alpha$ a $\beta$. Posteriormente, a $994{ }^{\circ} \mathrm{C}$ y para ambas composiciones, se evidenció un pico exotérmico asociado a la transformación de metacaolín a espinela o a fase premullita. Finalmente, un pico endotérmico aproximadamente a los $1181^{\circ} \mathrm{C}$ fue observado en la mezcla con composición CTA $25 \%$, indicando posiblemente la formación de una fase líquida principalmente derivada de la ceniza.

La tabla III indica que en las cerámicas obtenidas a $1200^{\circ} \mathrm{C}$, a medida que se reemplazó el feldespato por CTA, hubo un aumento en el porcentaje de contracción lineal, siendo máximo para una adición del 50\% de CTA. A partir de aquí decreció, aunque la mezcla con CTA $100 \%$ presentó una contracción similar a la mezcla con CTA $0 \%$.

La densidad aparente de las piezas cocidas se incrementó con el porcentaje de adición de CTA en reemplazo de feldespato comparada con la mezcla patrón CTA $0 \%$, donde aquella mezcla CTA 25\% mostró la mayor densidad aparente, siendo decreciente para mayores adiciones de CTA; incluso, para aquellas mezclas con CTA $100 \%$ existió una disminución de la densidad aparente hasta ser menor a la de la mezcla patrón. Los porcentajes de porosidad aparente, porosidad total y absorción de agua de las piezas cocidas mostraron tendencias similares. Para las piezas cocidas CTA $25 \%$, fueron 0,$88 ; 0,84$ y 0,83 veces menor, respectivamente, en comparación con la mezcla patrón CTA $0 \%$. Lo anterior, es atribuido a la formación de la fase líquida que tiende a acercar las partículas y, por consiguiente, la porosidad abierta disminuye; sin embargo, para aquellas mezclas con CTA $100 \%$ hubo un incremento en estos de 1,23; 1,12 y 1,32 veces, respectivamente con respecto a CTA $0 \%$. Cabe mencionar que la CTA 75\% mostró claramente el efecto de la reducción de la contracción y el incremento de la porosidad (ver tabla III), comportamiento típico del fenómeno de hinchamiento (swelling ó bloating) presente durante la sinterización en fase

TABLA III. PROPIEDADES FísICAS DE LAS MUESTRAS DE CERÁMICA OBTENIDAS MEDIANTE EL PROCESO DE COCCIÓN.

\begin{tabular}{|c|c|c|c|c|c|}
\hline Propiedades físicas & CTA 0\% & CTA 25\% & CTA 50\% & CTA 75\% & CTA 100\% \\
\hline Contracción lineal $(\%)$ & 4,70 & 6,31 & 6,76 & 6,44 & 4,75 \\
\hline Densidad aparente $\left(\mathbf{g} / \mathbf{c m}^{3}\right)$ & 1,84 & 1,95 & 1,88 & 1,87 & 1,72 \\
\hline Porosidad aparente (\%) & 20,15 & 17,69 & 17,84 & 24,52 & 29,79 \\
\hline Porosidad total (\%) & 26,65 & 22,40 & 24,51 & 10,45 & 14,41 \\
\hline Absorción de agua (\%) & 10,94 & 9,08 & 9,49 & 2,49 & 2,45 \\
\hline Densidad real $\left(\mathbf{g} / \mathbf{c m}^{3}\right)$ & 2,51 & 2,51 & 2,49 & & 24 \\
\hline
\end{tabular}


líquida de cerámicas tradicionales. Lo anterior puede ser atribuido a que con el incremento en la proporción de CTA, mayor es el contenido de sustancia generadora de gases $(\mathrm{KCl})$ y aumenta la cantidad de fase líquida de origen potásico. La densidad real de las piezas cocidas se redujo para valores superiores al 25\% de CTA en reemplazo de feldespato.

Braganca y Bergmann (2004) mencionan que la densidad aparente es una propiedad que tiene una fuerte influencia sobre el módulo de ruptura; por lo que generalmente a mayor densidad aparente se presenta mayor módulo de ruptura [14]; en confirmación a lo anterior, al observar en la figura 5 la resistencia a la compresión de las piezas cocidas, se evidencia una tendencia similar a los resultados obtenidos en la densidad aparente. De esta manera, la CTA 25\% generó la mayor resistencia a la compresión siendo 2,1 veces mayor en comparación con la mezcla patrón CTA 0\%; sin embargo, a partir de adiciones posteriores de CTA decreció la resistencia a la compresión, así para las mezclas con CTA 100\% la resistencia a la compresión fue 0,74 veces menor que la de CTA $0 \%$.

Con base en la absorción de agua (10-15\%), las cerámicas CTA 0\%, CTA 75\% y CTA 100\% obtenidas, podrían ser consideradas como lozas finas con posibles aplicaciones artísticas y de cocina, entre otras; incluso, aquellas cerámicas con CTA 25\% y CTA 50\% podrían ser consideradas como lozas semivítreas para vajillería ó artísticas (absorción de agua del 4 - 9\%) [22].

Las micrografías de MEB en modo BES (electrones retrodispersados, por sus siglas en inglés) (ver figura 6 (a y

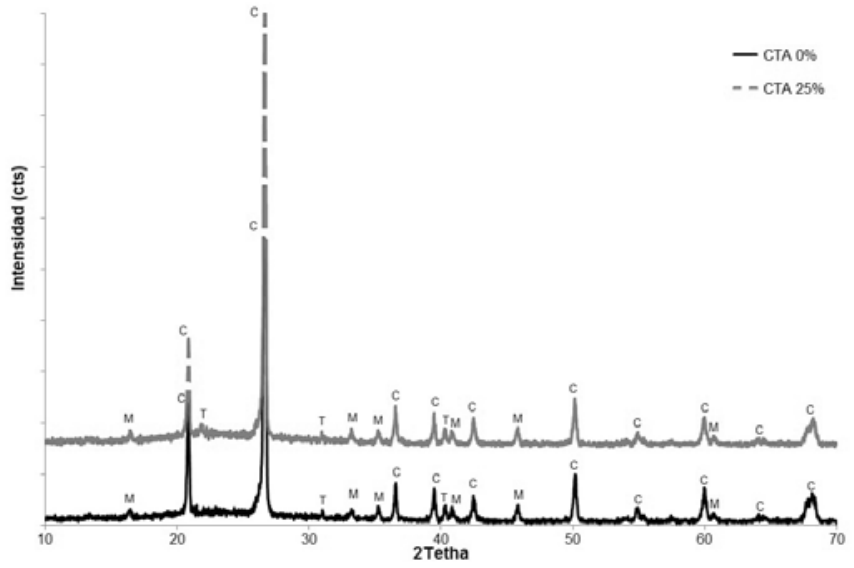

Figura 7. Patrones de DRX de probetas CTA $0 \%$ y CTA $25 \%$ cocidas, ( $\mathrm{M}=$ mullita, $\mathrm{C}=$ cuarzo, $\mathrm{T}=$ tridimita $)$.

b)), muestran una superficie similar tanto para las cerámicas cocidas CTA $0 \%$ como para la CTA $25 \%$.

Las micrografías de MEB en modo SEI (electrones secundarios, por sus siglas en inglés) (ver figura 6 (c, d y e, f)), evidenciaron en la microestructura de ambas piezas cocidas (CTA $0 \%$ y CTA 25\%) la presencia de cristales de mullita primaria y secundaria (agujas elongadas); donde se aprecia mayor densificación en las muestras CTA 25\%. Estos resultados fueron corroborados con los ensayos de DRX de las muestras CTA $0 \%$ y CTA 25\% (ver figura 7), evidenciando la presencia de

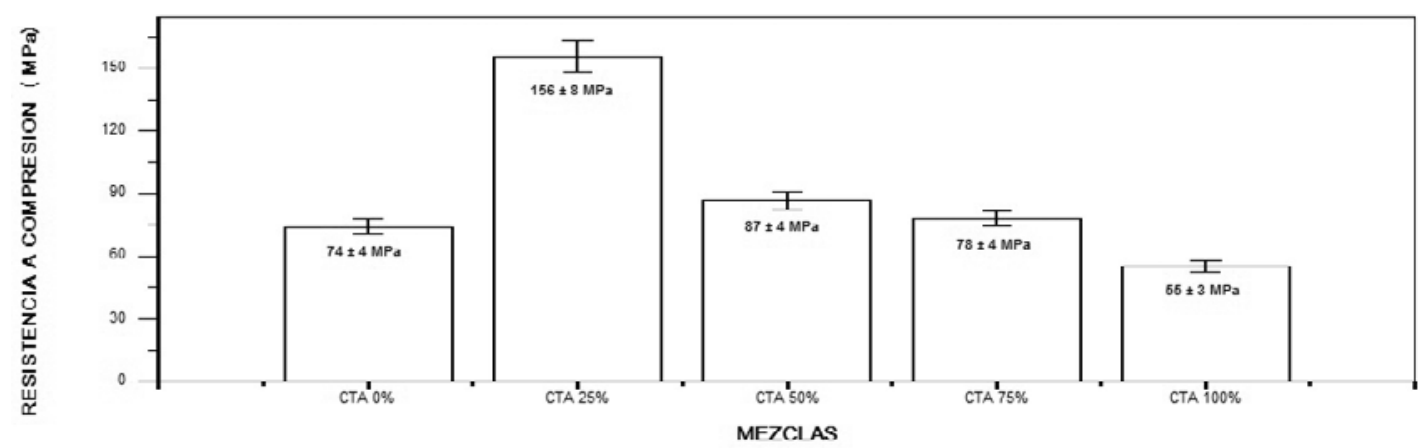

Figura 5. Resistencia a la compresión de las muestras de cerámica obtenidas mediante el proceso de cocción.



Figura 6. Micrografías MEB en modo BES de superficies pulidas de probetas CTA $0 \%$ (a) y CTA 25\% (b); y micrografías MEB en modo SEI de superficies pulidas de probetas CTA $0 \%$ (c y d) y CTA 25\% (e y f) cocidas. 
la fase mullita $\left(2 \theta=16,45^{\circ} ; 33,26^{\circ} ; 35,31^{\circ}\right.$ y $\left.40,91^{\circ}\right)\left(3 \mathrm{Al}_{2} \mathrm{O}_{3} 2 \mathrm{SiO}_{2}\right)$ (ICSD 99330), responsable, en parte, de la resistencia mecánica de las porcelanas; además de la presencia de otras fases como cuarzo bajo $\left(2 \theta=26,67^{\circ}\right.$ y $\left.20,88^{\circ}\right)\left(\mathrm{SiO}_{2}\right)(\mathrm{ICSD} 41447)$ y tridimita $\left(2 \theta=21,73^{\circ} ; 30,19^{\circ}\right.$ y $\left.40,3^{\circ}\right)\left(\mathrm{SiO}_{2}\right)$ (ICSD 1109). Cabe anotar, que el contenido de la fase mullita es muy escaso a causa del bajo contenido de caolinita en los materiales de partida.

\section{CONCLUSIONES}

Con base en los resultados obtenidos se concluye que la ceniza de tamo de arroz es un posible reemplazo parcial del feldespato en la elaboración de lozas cerámicas; siendo posible obtener una microestructura correspondiente a una porcelana (granos de cuarzo y agujas de mullita primaria y secundaria en una matriz vítrea feldespática) mediante la adición de CTA. Las piezas cocidas con $25 \%$ de CTA reportan un valor de densidad real similar a la obtenida por la pieza cocida de una mezcla patrón con el $100 \%$ de feldespato. Los porcentajes de porosidad aparente, porosidad total y absorción de agua disminuyen con sustituciones hasta de un 75\% del feldespato por la CTA, en comparación con la mezcla patrón. No obstante, para valores de sustitución superiores al $50 \%$, se observa con claridad el fenómeno de hinchamiento típico del proceso de sinterización en presencia de fase líquida de las cerámicas tradicionales. Igualmente, los valores de resistencia a la compresión de estas piezas cocidas aumentan con adiciones de CTA de hasta un $75 \%$ en reemplazo del feldespato y se duplican con referencia a los de la pieza cocida sin adición cuando la sustitución es del $25 \%$.

\section{AGRADECIMIENTOS}

Los autores quieren agradecer a la Universidad del Valle (Colombia), al Instituto de Tecnología Cerámica (ITC), al Instituto de Tecnología de Materiales de la Universidad Politécnica de Valencia, al CENM y a COLCIENCIAS por el apoyo brindado para el desarrollo de este estudio. Se agradece a ECOS-Nord/COLCIENCIAS/ICFES/ICETEX por el apoyo a la Acción ECOS-Nord No. C09P01, en la cual se hicieron algunos ensayos de esta investigación. En particular, en esta publicación se presentan los resultados parciales de la investigación "Cerámicas Triaxiales basadas en Cenizas del Tamo de Arroz" código 110652128358 apoyada por COLCIENCIAS, Convocatoria 521 de 2010, contrato RC. No. 325-2011.

\section{REFERENCIAS}

(1) Karimi, K.; Emtiazi, G.; Taherzadeh, M.J. Production of ethanol and mycelial biomass from rice straw hemicellulose hydrolyzate by Mucor indicus. Process Biochem., 41(3) 653-658, 2006.

(2) Cuevas, M.A. El tamo del arroz: El subproducto de la sostenibilidad, Revista Arroz, [online], 2008. [Consulta: 2009-04-01] <http://www. fedearroz.com.co/arroz/448/resumen.shtml >.

(3) Ministerio del Medio Ambiente. Guía Ambiental del Arroz. [pdf] Sociedad de Agricultores de Colombia - SAC, Federación Nacional de Arroceros - FEDEARROZ, 2005. [Consulta: 2009-04-01] <http://www. siame.gov.co/siame/documentos/Guias_Ambientales/Gu\%C3\%ADas $\% 20$ Resoluci $\%$ C3\%B3n $\% 201023 \% 20$ del $\% 2028 \% 20$ de $\% 20$ julio $\% 20$ de $\% 20$ 2005/A GRICOLA $\% 20$ Y $\% 20$ PECUARIO/Guia $\% 20$ Ambiental $\% 20$ para $\% 20$ el\%20subsector \%20arrocero.pdf $>$.

(4) Montoya, J.C. Hongos: Riqueza de la Naturaleza Poco Explotada, [online] 1997. [Consulta: 2009-04-01] <http://aupec.univalle.edu.co/informes/ abril97/boletin34/hongos.html>.

(5) Kadam, K.L.; Forrest, L.H.; Jacobson, W.A. Rice straw as a lignocellulosic resource: collection, processing, transportation, and environmental aspects. Biomass Bioenergy., 18(5) 369-389, 2000.

(6) Hiziroglu, S.; Jarusombuti, S.; Bauchongkol, P.; Fueangvivat, V. Overlaying properties of fiberboard manufactured from bamboo and rice straw. Ind. Crops Prod., 28(1) 107-111, 2008.

(7) Rodríguez, A.; Moral, A.; Serrano, L.; Labidi, J.; Jiménez, L. Rice straw pulp obtained by using various methods. Bioresour. Technol., 99(8) 2881-2886, 2008.

(8) Martínez, H.J.; Acevedo, X.; Espinal, C.F. La Cadena del Arroz en Colombia - Una Mirada Global de su Estructura y Dinámica 1991 - 2005. [pdf] Ministerio de Agricultura y Desarrollo Rural Observatorio Agrocadenas Colombia (Documento No. 89), 2005. [Consulta: 2009-04-01] <http:// www.agrocadenas.gov.co/arroz/documentos/caracterizacion_arroz. pdf $>$.

(9) DANE. Encuesta Nacional de Arroz Mecanizado - II Semestre. Boletín de Prensa [pdf], 2010. [Consulta: 2011-03-07] <http:/ / www.dane.gov.co/ files/investigaciones/boletines/arroz/bol_arroz_IIsem10.pdf>.

(10) Nguyen, X.T. The need for improved utilization of rice straw as feed for ruminants in Vietnam: An overview. Livestock Research for Rural Development., [online], 10(2), 1998. [Consulta: 2008-07-10] <http://www. cipav.org.co/lrrd/1rrd10/2/trach102.htm>.

(11) Carty, W.M.; Senapati, U. Porcelain-Raw materials, processing, phase evolution and mechanical behavior. J. Am. Ceram. Soc., 81(1) 3-20, 1998.

(12) Motta, J.F.; Cabral, Jr. M.; Tanno, L.C.; Zanardo, A. As matérias primas cerâmicas. Parte II: Os minerais industriais e as massas da cerámica tradicional, Revista Cerâmica Industrial., 7(1) 33-40, 2002.

(13) Correia, S.L.; Oliveira, A.P.; Hotza, D.; Segadaes, A.M. Properties of triaxial porcelain bodies: Interpretation of statistical modeling. J. Am. Ceram. Soc., 89(11) 3356-3365, 2006.

(14) Braganca, S.R.; Bergmann, C.P. Traditional and glass powder porcelain: technical and microstructure analysis. J. Eur. Ceram. Soc., 24(8) 2383-2388, 2004.

(15 Andreola, F.; Barbieri, L.; Karamanova, E.; Lancellotti, I.; Pelino, M. Recycling of CRT panel glass as fluxing agent in the porcelain stoneware tile production. Ceram. Int., 34(5) 1289-1295, 2008.

(16) Dana, K.; Das, S.K. Partial substitution of feldspar by B.F slag in triaxial porcelain: Phase and microstructural evolution. J. Eur. Ceram. Soc., 24(1516) 3833-3839, 2004.

(17) Jenkins, B.M.; Baxter, L.L.; Miles Jr, T.R.; Miles, T.R. Combustion properties of biomass. Fuel Process. Technol., 54(1-3) 17-46, 1998.

(18) Thy, P.; Jenkins, B.M.; Grundvig, S.; Shiraki, R.; Lesher, C.E. High temperature elemental losses and mineralogical changes in common biomass ashes. Fuel., 85(5-6) 783-795, 2006.

(19) Braganca, S.R.; Bergmann, C.P.; Hubner, H. Effect of quartz particle size on the strength of triaxial porcelain. J. Eur. Ceram. Soc., 26(16) 3761-3768, 2006.

(20) Mattyasovszky-Zsolnay, L. Mechanical strength of porcelain. J. Am. Ceram. Soc., 40(9) 299-306, 1957.

(21) Stathis, G.; Ekonomakou, A.; Stournaras, C.J.; Ftikos, C. Effect of firing conditions, filler grain size and quartz content on bending strength and physical properties of sanitaryware porcelain. J. Eur. Ceram. Soc., 24(8) 2357-2366, 2004.

(22) Kreith, F. Mechanical Engineering Handbook (Frank Kreith). CRC Press LLC, Boca Raton, 85-102, 1999. 